\title{
EMOTLAB: Software for studying emotional signaling in economic bargaining games
}

\author{
TIMOTHY KETELAAR \\ New Mexico State University, Las Cruces, New Mexico \\ Ben Preston \\ Houston, Texas \\ Deborah RuSSELL \\ New Mexico State University, Las Cruces, New Mexico \\ Mark DaVIS \\ University of West Alabama, Livingston, Alabama \\ AND \\ Garrett Strosser \\ New Mexico State University, Las Cruces, New Mexico
}

\begin{abstract}
EMOTLAB software creates a virtual social environment in which individuals interact via computer with a virtual interaction partner in a series of economic bargaining games. The virtual partner appears on the participant's computer screen as a digital image (e.g., video or picture file) during each trial. A key feature of EMOTLAB software is its ability to control both the strategic behavior and the emotion signaling behavior (e.g., anger vs. embarrassment) of the virtual interaction partner. By simply editing a series of text files that control the subroutines governing the different features of the experiment (payoff structure, number of trials, etc.), EMOTLAB can generate an essentially infinite number of different social bargaining situations in which participants earn monetary payoffs contingent upon their decisions. This paper provides an overview of this software and how one can edit various subroutines to generate a typical experimental session in which research participants encounter a virtual interaction partner who displays different emotional signals.
\end{abstract}

Many economists believe that emotions can exert a powerful influence on economic decisions (Elster, 1995, 2000; Frank, 1988, 2004; Hirschleifer, 1987, 2001). Unfortunately, in the behavioral economics literature it is often the case that the role of emotions is theoretically explored and then empirically ignored (see Ketelaar, 2004, 2006). Economist Robert Frank (1988), for example, developed a theoretical argument for why individuals who experience feelings of guilt while contemplating cheating will often overcome the temptation to cheat. Frank (1988) argued that guilt-prone individuals forgo opportunities to cheat, not because they fear getting caught, but rather because their feelings of guilt effectively alter the payoffs that they face. According to Frank (1988), an individual experiencing a strong emotion is literally compelled to view strategic options in terms of their long-term, rather than short-term, consequences (see also Smith, 1759/2000). Thus, a decision-maker who experiences a negative emotion, such as guilt, while contemplating cheating will correctly perceive the long-term negative consequences of pursuing such temptations. In this manner, Frank (1988) argues that emotions can generate seemingly irrational decisions because emotions often provoke individuals to maximize long-term payoffs at the expense of forgoing immediate rewards (see also Hirschleifer, 1987, 2001; Schelling, 1960, 1984). Unfortunately, as is the case for many game theoretic explanations of emotional decision-making, Frank's (1988) model has inspired more citations than empirical tests.

Fortunately, there is a now a small but growing body of empirical research examining the strategic role of emotions in economic decision-making (see Ketelaar, 2006, and Zeelenberg \& Pieters, 2006 for reviews). In this regard, several studies suggest that participants in economic bargaining games wear their strategic dispositions not on their sleeves, but on their faces (see Yamagishi, Tanida, Mashima, Shimoa, \& Kanazawa, 2003). In one study, Frank, Gillovich, and Regan (1993) found that participants who interacted with a randomly assigned person for only 30 min could accurately predict how often this person would cooperate in a subsequent social bargaining game known as the repeated

T. Ketelaar, ketelaar@nmsu.edu 
prisoner's dilemma. Although this study did not reveal the specific nonverbal cues that predicted an individual's tendency to cooperate or defect, several recent studies point to facial displays of emotion as a prime candidate for these nonverbal signals of strategic dispositions. In one study, participants who viewed videotapes of individuals interacting with another person were later able to accurately discriminate altruists from nonaltruists ${ }^{1}$ by simply taking into account whether the individual displayed involuntary happiness smiles (Brown, Palameta, \& Moore, 2003; see also Brown \& Moore, 2002). ${ }^{2}$ Although game theorists assume that these sorts of nonverbal signals (e.g., smiles) will often be ignored as cheap talk (Searcy \& Nowicki, 2003), one experimental economics study found that smile displays routinely evoked attributions of cooperativeness as well as behavioral acts of trust in a bargaining game known as an extensive form trust game. ${ }^{3}$ When participants were presented with a photograph of their alleged counterpart, participants chose to trust a smiling partner $68 \%$ of the time, but trusted a nonsmiling partner only $55 \%$ of the time (Scharlemann, Eckel, \& Wilson, 1999). While these early findings are encouraging, the experimental economics convention of studying anonymous pairings of strangers who never directly interact (except perhaps via computer) has made it difficult to incorporate face-to-face emotional experiences into game theoretic models of economic behavior. In this regard, EMOTLAB software is designed to facilitate empirical research on emotion and economic decision-making by providing a platform for manipulating and measuring emotional signals in social bargaining games.

\section{GOALS AND DEVELOPMENT OF EMOTLAB SOFTWARE}

EMOTLAB allows the researcher to generate a virtual social environment in which participants interact via computer with a virtual interaction partner in a series of economic bargaining games. The virtual partner appears on the participant's computer screen as a digital image during each trial. A key feature of EMOTLAB is its ability to control both the strategic behavior and emotion signaling behavior (e.g., anger versus embarrassment) of this virtual interaction partner. The behavior of the virtual opponent can be easily "programmed" by the researcher without requiring extensive computer programming experience or expertise. By simply editing a series of text files that control the subroutines governing the different features of the experiment (payoff structure, number of trials, behavior of the virtual partner, etc.) EMOTLAB can generate a large variety of different social bargaining situations in which participants earn monetary payoffs contingent upon their decisions. The next section of this paper provides a brief rationale for studying interactions with virtual partners in economic bargaining games. The remainder of the paper provides a quick overview of this software and how one can edit the various subroutines to generate a typical experimental session in which research participants encounter a virtual social interaction partner who displays different emotional signals in a series of economic bargaining games.

\section{USING VIRTUAL PARTNERS TO STUDY SOCIAL DECISION-MAKING}

Exploring how individuals utilize information contained in the emotional displays that can occur during social interactions is an important question for both psychologists and behavioral economists (see Fridlund, 1994; Ketelaar, 2004, 2006). Emotion researchers agree that there are at least six distinct, socially meaningful facial displays associated with specific emotional states and behavioral tendencies (Ekman, 2003; Fridlund, 1994). These include facial displays associated with happiness, embarrassment, contempt, anger, and disgust, as well as the familiar neutral face (see Figure 2). Each of these displays corresponds to a specific pattern of facial muscle movements that is highly specific in terms of the pattern of onset, offset, and coordination among muscle actions (Ekman, Friesen, \& Hager, 2002). Unfortunately, naturally occurring emotion displays are difficult to capture and measure during "real time" face-to-face economic transactions and manipulating facial displays in a systematic and ecologically valid fashion (via trained actors) is often not a realistic methodological option in most laboratory settings. In regards to the possibility of employing trained actors to systematically manipulate the emotion displays of an interaction partner, it must be noted that the facial musculature is innervated by both voluntary and involuntary pathways of the seventh cranial nerve. As a result, many facial displays of emotion are comprised of patterns of muscular onset, offset, and coordination that are not under voluntary control. ${ }^{4}$ In fact, some have argued that the ability to spontaneously and accurately generate the appropriate onset, offset, and coordination of specific muscle movements comprising particular emotion displays is restricted to a small set of facial "gymnasts" (Ekman, 2003). For example, the most widely used system for training in facial muscle movement and measurement - the so-called Facial Action Coding system (FACs) - requires approximately 80 hours of training before raters are able to systematically and reliably identify the individual muscle movements that comprise each emotion (see Ekman, Friesen, \& Hager, 2002). Moreover, as trained FACs coders already know, the ability to reliably detect specific facial muscle movements in others is often independent of the ability to spontaneously and voluntarily generate these same patterns on one's own face. With these pragmatic concerns in mind, EMOTLAB was designed to facilitate the study of emotional signaling in strategic social interactions by enabling the experimenter to systematically manipulate the emotional signaling behavior of a virtual social interaction partner.

\section{HOW EMOTLAB'S VIRTUAL PARTNER WORKS}

A key feature of EMOTLAB is the virtual interaction partner that can be programmed to display a variety of emotional signals and strategic behaviors in a vast number of economic bargaining games. The virtual partner takes the form of a digital image (movie or picture file) that appears on the "results" screen that is generated after each trial (see Figure 1). Immediately after the participant has 
A

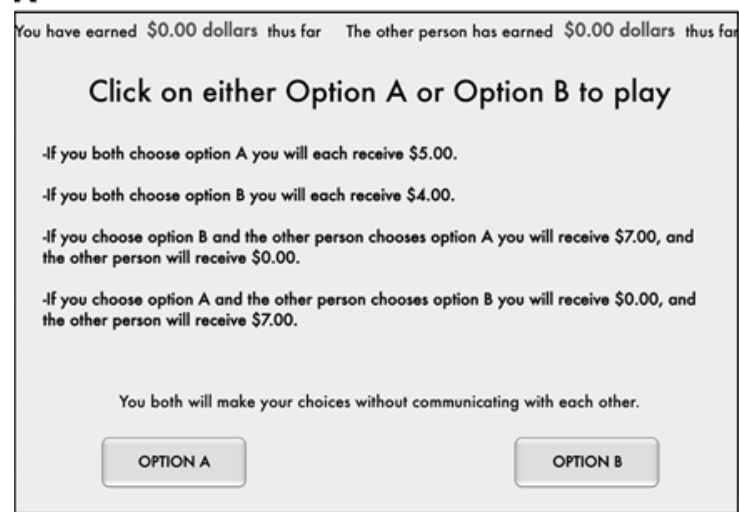

C

You have earned $\$ 7.00$ dollars thus for The other person has earned $\$ 0.00$ dollars thus for

\section{Results:}

You chose option B.

The other person chose option $A$.

You get $\$ 7.00$ and the other person gets $\$ 0.00$.

\section{Click Here to Continue}

$\mathbf{E}$

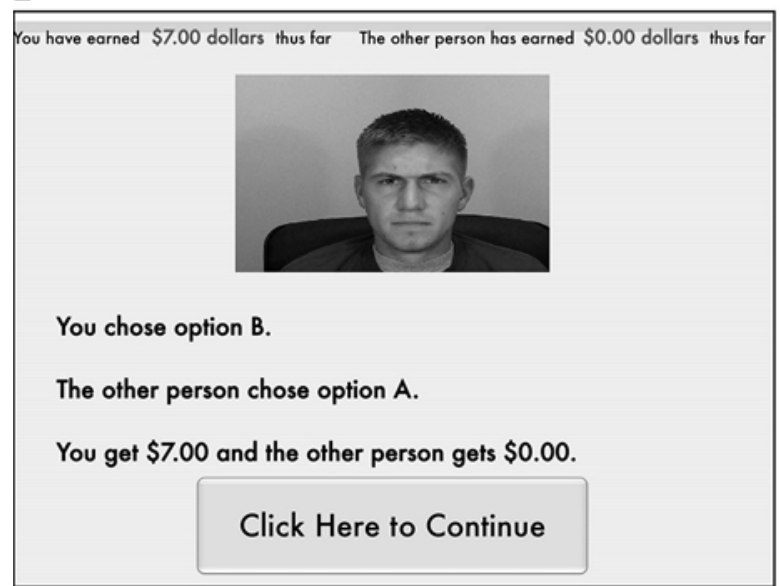

B

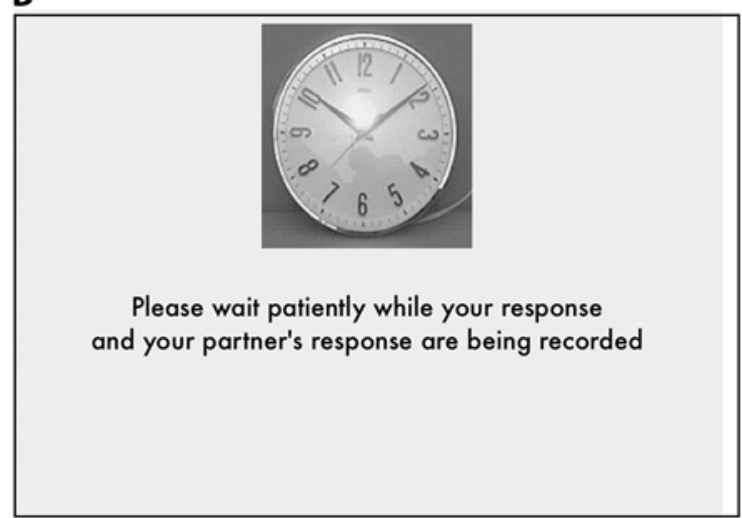

D

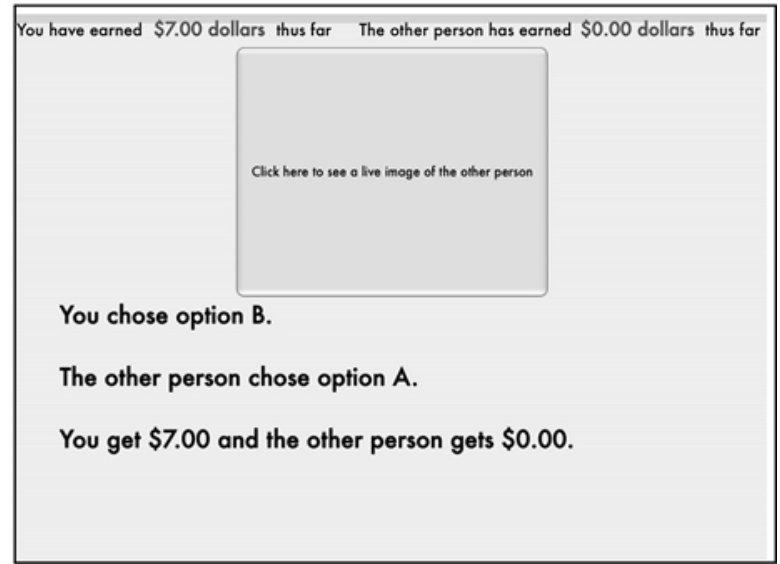

$\mathbf{F}$

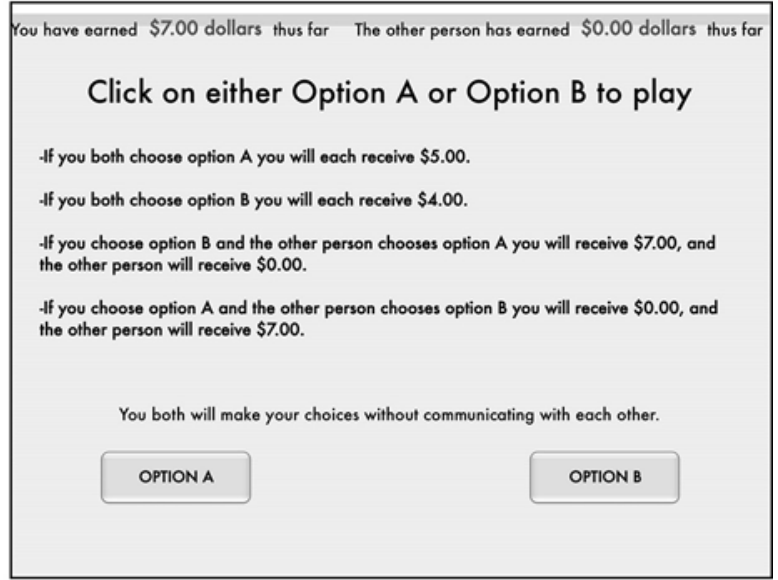

Figure 1. Screen captures depicting how the virtual partner's image is displayed on the results screen.

made their strategy choice (e.g., selecting "Option A" or "Option B," see Figure 1A), a screen immediately appears that instructs participants to wait patiently while the participant's response and the response of the partner are being recorded (see Figure 1B). This "wait patiently" screen is set on a random delay ranging from 0 to $5 \mathrm{sec}$ to mimic computer network traffic. ${ }^{5}$ Immediately after this "wait patiently" screen disappears, an initial results screen appears (see Figure 1C) and announces the strategy choices (option A or B) selected by each participant, along with a summary of the monetary amounts accrued by each participant during this particular trial. At the top of the screen a running total is displayed which keeps track of the total monetary earnings accrued for each participant across all trials played thus far. When the participant clicks on the "Click here to continue" button at the bottom of the screen (see Figure 1C), a large button appears at the top of the screen (see Figure 1D). This button informs participants to 
"Click here to see a live image of the other person." When the participant clicks on this button, the virtual partner appears (see Figure 1E) on the screen as a digital image (e.g., movie or picture). After viewing this image, the participant can then click on a button labeled "Click here to continue" (Figure 1D) which allows the participant to proceed to the next trial (Figure 1E). In this manner, the participant can interact with a virtual partner in a series of economic bargaining games, with the opportunity to view the partner's reaction (emotion display) immediately after observing the results of each trial.

EMOTLAB can be presented to participants as a program that displays images of "virtual" people that are stored in the computer, or as a program that - in conjunction with the appropriate cover story — ostensibly permits communicating with a "real" person in real time. ${ }^{6}$ The program is currently distributed with image files that allow a researcher to select from 2 different virtual partners (1 male, 1 female) each of whom is capable of displaying six distinct emotion signals. ${ }^{7}$ Figure 2 displays a sample of these images from the two virtual partners including sample images of the five emotion displays corresponding to anger, contempt, disgust, embarrassment, happiness, and the neutral display. In order to insure that each emotion image depicts the appropriate pattern of muscle and head movements associated with each specific emotion display (see Ekman, 2003), each image file was scored by 2 coders (all Kappas $>0.60$ ) trained in the Facial Action Coding system (see Ekman et al., 2002). All image files are stored in the Movies folder located in the main EMOTLAB folder.
Several variations of each neutral display image (movie or picture) are provided for each virtual partner (see Figure 2) because pilot testing suggested that when EMOTLAB is presented as a program that ostensibly permits communicating with a real person in real time, participants are more likely to believe that they are interacting with an actual person (as opposed to a virtual partner) when slightly different variations of the neutral display are employed across several consecutive trials. To facilitate using EMOTLAB as a program that ostensibly permits communicating with a real person in real time, the experimenter can also connect a digital video camcorder to the computer and inform participants that "your partner will be able to view live images of you, just as you are able to view live images of them."

Regardless of whether the experimenter wishes to employ a cover story regarding interacting with "real persons in real time," EMOTLAB is explicitly designed to allow the experimenter to use a video camcorder to capture the participant's online behavior during certain key events that that occur during each trial of the virtual social interaction. Specifically, EMOTLAB comes preequipped with a series of embedded audio tones that demarcate the important screen transitions (e.g., the appearance of the results screen, the appearance of the image of the virtual partner, etc.; see Figure 1) during each trial. When the computer is connected via an audio cable to a digital video camcorder, these tones are embedded on the audio track of the video in a manner that enables the researcher to capture the behavior of the participant during these key events. This feature can be disabled by simply turning off the volume on your computer.

\section{Two Virtual Partners}
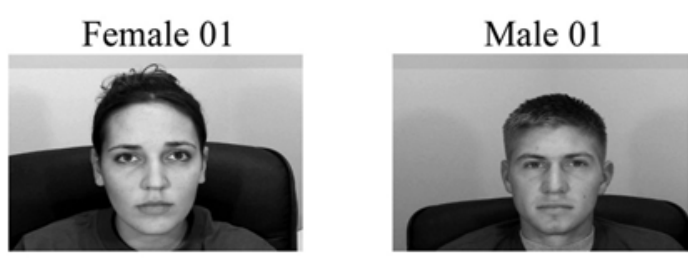

\section{A sample of the Five Emotion Displays (including different neutral displays)}

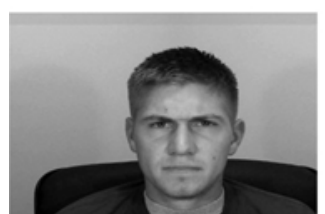

male01Anger01.jpg

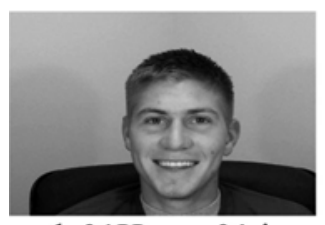

male01Happy01.jpg

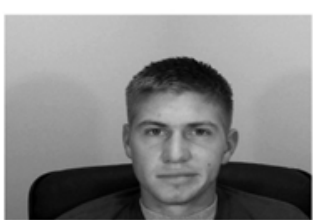

male01Contempt.jpg

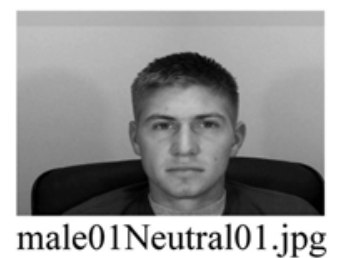

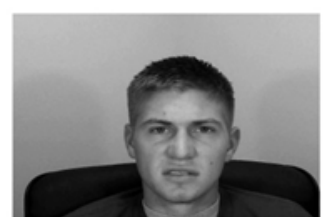

male01Disgust01.jpg

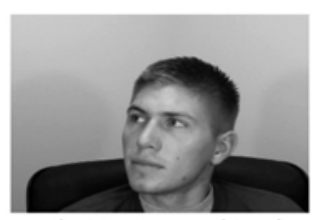

male01Neutral02.jpg

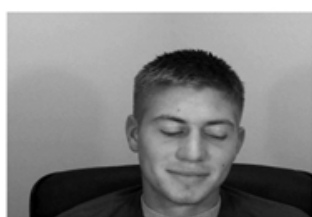

male01Embarrass01.jpg

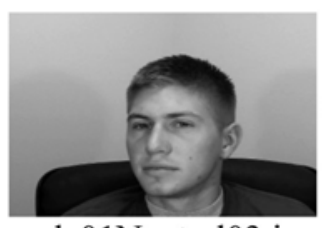

male01Neutral03.jpg

Figure 2. Digital images depicting emotional signals displayed by the virtual interaction partner. 


\section{BASIC FEATURES OF EMOTLAB SOFTWARE}

Behavioral economics is that branch of economics that studies strategic behavior in social interactions that can be modeled as "games" (Camerer, 2003). From the perspective of formal "game theory" (von Neumann \& Morgenstern, 1944) a game corresponds to any social interaction in which a participant's outcomes (e.g., their monetary earnings) are dependent upon not only their own behavior (what they elect to do), but also the behavior of their interaction partner (what their opponent elects to do). In this context, hitting a tennis ball against a wall does not constitute a "game" because a participant's outcomes are dependent solely upon his or her own behavior (e.g., the wall does not emit behavior in response to the participant). By contrast, social interactions in which the monetary earnings accrued by one participant are dependent upon not only their own strategy choices, but also the strategy choices of their interaction partner, are properly referred to as games. In this regard, EMOTLAB provides a platform for constructing a vast number of 2 (person) $\times 2$ (alternative) economic bargaining games in which participants interact with a virtual opponent.

The core logic of the EMOTLAB program is contained in a series of subroutines known as PMX files (see Figure 3). "PMX" is an abbreviation for "payoff matrix." A PMX file is essentially an editable text file that specifies the parameters of play for one trial and can be created from scratch or by simply copying and editing an existing PMX file. For the Windows environment, the "Wordpad" text editor can be used to create and modify these files. For Macintosh users, Mac OS X includes a suitable text editor called "TextEdit." EMOTLAB Version 1.0 comes preequipped with two PMX files: "MovieDemo.pmx" and "PhotoDemo.pmx." Editing a PMX file allows the experimenter to control the following four features of game play for a specific trial: (1) the payoff matrix; (2) the emotional signal (facial display of emotion) that is generated by the virtual partner; (3) the strategic behavior (e.g., Option A or B) that is selected by the virtual partner; and (4) whether a prediction screen will appear during a particular trial. The following sections contain a very brief description of each of these four features of game play that can be programmed by editing a PMX file.

\section{Specifying the Payoff Matrix}

EMOTLAB software is designed to study behavior in economic bargaining games in which participants are paid real money contingent upon their actual performance. ${ }^{8}$ The pattern of monetary outcomes generated during each trial of an EMOTLAB experiment corresponds to the generic "payoff matrix" displayed in Figure 4. A payoff matrix is simply a tabular depiction of the interdependent pattern of outcomes that allows us to identify a social interaction as a economic bargaining game. The variables $\$ \mathrm{X}, \$ \mathrm{Y}$, \$Z, etc., depicted in Figure 4 correspond to the various monetary earnings that the participant would earn each trial (round of play) for each of the four possible outcomes that can occur in a 2 (person) $\times 2$ (alternative) game. For example, if both the column player (participant) and the row player (virtual opponent) select Option A, the participant would earn $\$ \mathrm{X}$ and the virtual partner would earn \$Y (see Figure 4). Alternatively, if the participant selected Option B and the virtual partner selected Option A, the participant would earn $\$ Z$ and the virtual partner would earn \$P (see Figure 4).

The actual dollar amounts that are earned by participants - corresponding to the variables $\$ \mathrm{X}, \$ \mathrm{Y}, \$ \mathrm{Z}$, etc., in the generic payoff matrix depicted in Figure 4-must be specified by the experimenter in the first two lines of text in the PMX file (see Figure 3). All values in the first two lines of a PMX file refer to dollars, and EMOTLAB allows these monetary amounts to be presented as decimals (e.g., $\$ 5.50$, $\$ 7.75$ ) as well as whole numbers (e.g., \$5, \$7). For example, in "MovieDemo.pmx," the first two lines of text (see Figure $5 \mathrm{~A}$ ) specify that the participant and the virtual partner will both earn $\$ 5$ if they both select Option A during a given trial (see Figure 5A) and alternatively that the participant will earn $\$ 7$ and the virtual partner will earn $\$ 0$ if the participant selects Option B and the virtual partner selects Option A. During each game-play trial in which this PMX file is used, the payoff matrix specified in the first two lines of the PMX file is displayed on the computer screen just prior to the participants' making their strategy choice (Option A or Option B). For example, the payoff matrix specified by "MovieDemo.pmx" (see Figure 3) is displayed as it would appear on the participant's computer screen in Figure 1A. Because the experimenter can specify up to eight distinct payoff outcomes (e.g, the variables $X$ through $T$ in Figure 4) across the four cells of the payoff matrix, EMOTLAB can generate up to 78 distinct 2 (person) $\times 2$ (alternative) economic bargaining games (see below).

The payoff matrix specified in "MovieDemo.pmx," for example, corresponds to a classic "prisoner's dilemma" game where Option B represents the optimal, or equilibrium, strategy. In this particular payoff environment (i.e., the prisoner's dilemma), selecting Option A when your opponent also selects Option A can be interpreted as an act of "mutual cooperation," whereas, selecting Option B when your opponent has selected Option A can be interpreted as an act of "defection" or "noncooperation" (see Poundstone, 1992). What makes this particular payoff environment a so-called "prisoners dilemma" (as opposed to some other game such as the "coordination game" or the "stag hunt") is the fact that the payoff matrix generated by "MovieDemo.pmx" satisfies a particular set of conditions regarding the rank ordering of the outcomes in the payoff matrix. For example, a prisoner's dilemma game corresponds to a payoff environment in which the following conditions are met: $\$ X=\$ Y, \$ S=\$ T, \$ Z=\$ R, \$ Q=\$ P$ and, more specifically, $S>(Z+Q) / 2$. Although the logic behind what distinguishes one $2 \times 2$ game (e.g., the prisoner's dilemma) from another (e.g., a coordination game) is beyond the scope of the present article, it can be noted that changing the payoff amounts in the payoff matrix can significantly alter the "meaning" of the various strategy alternatives - does Option A correspond to a cooperative response or an uncooperative response? - and thus, the nature of the game. The interested reader can consult the game theory literature to identify the types of games 


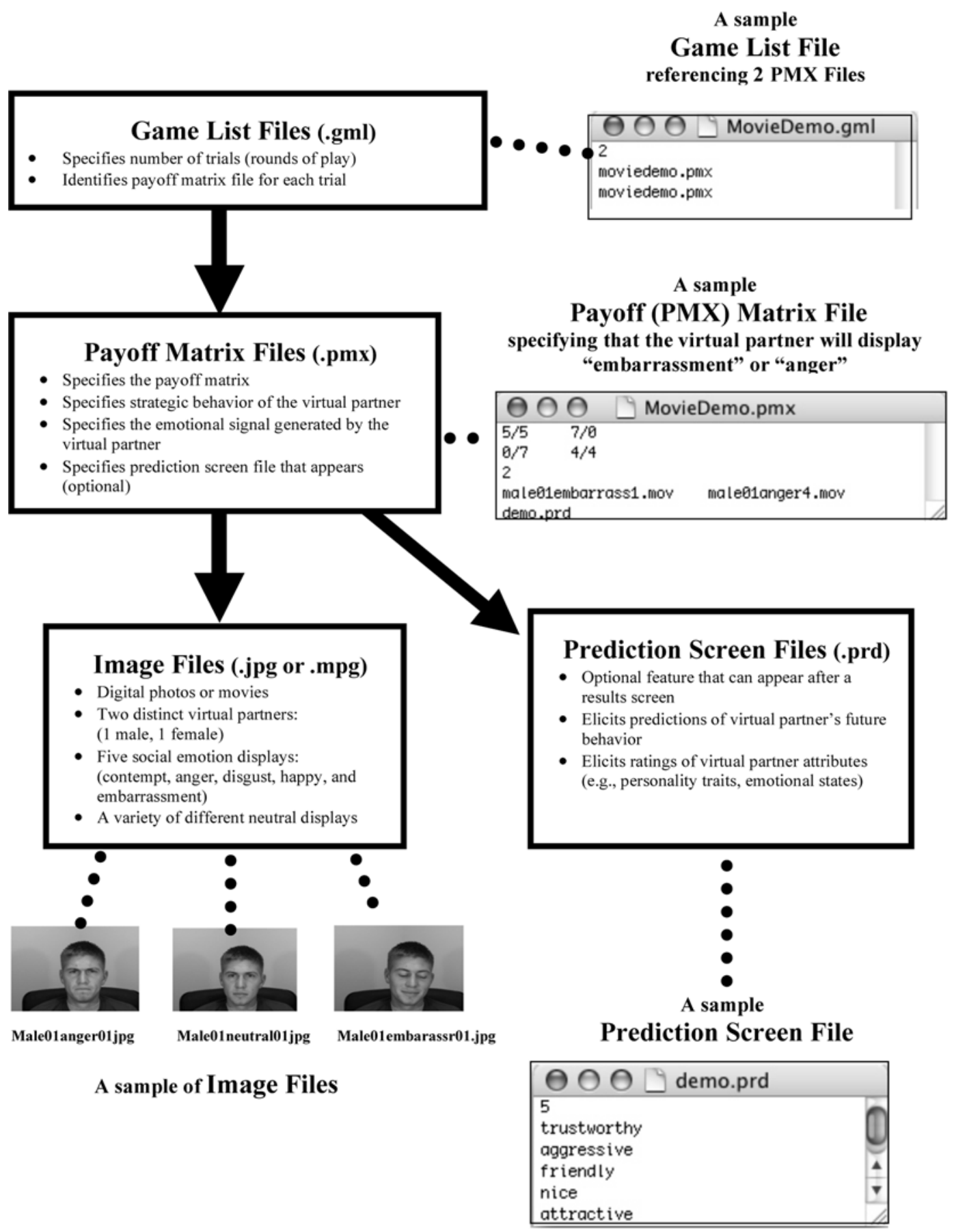

Figure 3. Hierarchical structure of EMOTLAB files.

(i.e., payoff environments) that best suit their particular research goals (see Hirshleifer, 2001 and Poundstone, 1992 for good introductions to the association between payoff environments and economic bargaining games).

\section{Specifying the Emotion Signaling Behavior of the Virtual Partner}

To control the emotional display of the virtual partner during a particular trial, the PMX file must reference the names of the particular image files (movies or photos) that will be displayed during that trial. The fourth line of text in a PMX File contains the names of these two image files (see Figure 5A). An important feature of EMOTLAB is that the software allows the experimenter to program the emotional signal of the virtual partner to be contingent upon the behavior of the research participant. The first image file listed on the left (see Figure 5A) specifies the digital image that will appear if the research participant 


\section{Player 1: \\ Participant's choice}

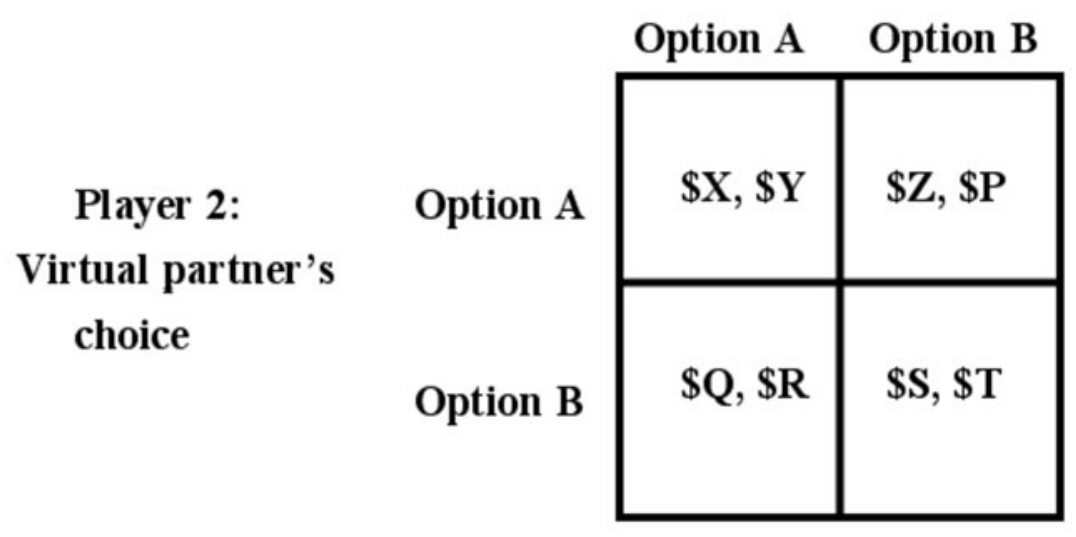

Note: The first value in each cell denotes the payoff to the column player (participant) and the second value denotes the payoff to the row player (virtual partner).

Figure 4. The generic payoff matrix for a single trial.

selects Option A during this trial. The second image file on the right specifies the digital image that will appear if the research participant selects Option B during this trial. In the example shown in Figure 5A, the PMX file (MovieDemo.pmx) specifies that the virtual partner will display the emotion signal of embarrassment if the research participant selects Option A (see Figure 5B); otherwise the virtual partner displays the emotion signal of anger if the research participant selects Option B (see Figure 5C). If both image files listed in the PMX file correspond to the same file name, the virtual partner will display the same image file regardless of which strategy option is selected by the participant. These image files must correspond to actual image files (movies or digital photos) stored in the Movies folder located in the main EMOTLAB folder.

\section{Specifying the Strategic Behavior of the Virtual Partner}

The third line of text in a PMX file specifies the strategic behavior of the virtual partner. EMOTLAB Version 1.0 comes preequipped with four distinct virtual partner strategies:

$$
\begin{aligned}
& 0=\text { The virtual partner plays Option A this trial. } \\
& 1=\text { The virtual partner plays Option B this trial. } \\
& 2=\text { The virtual partner plays Tit-for-Tat this trial. } \\
& 3=\text { The virtual partner plays Tat-for-Tit this trial. }
\end{aligned}
$$

As discussed in the previous section on "specifying the payoff matrix," the exact meaning of Option A and Option B are dependent upon the particular payoff environment generated by the PMX file. In the payoff environment specified by "MovieDemo.pmx" (a prisoner's dilemma game), Option A corresponds to a cooperative response and Option B corresponds to a noncooperative response; but if the experimenter were to alter the payoff environment (by changing values in the payoff matrix), this could significantly change what it means to select Option A as opposed to Option B. Again, the interested reader can consult the game theory literature to identify the types of games (i.e., payoff environments) that best suit their particular research goals.

Regardless of the payoff environment (specified by the payoff matrix), the experimenter can program two different virtual partner strategies ("tit-for-tat" and "tat-for-tit") that are contingent upon the behavior of the participant. If the experimenter indicates Strategy 2 in the fourth line of the PMX file (see Figure 5), this generates a contingent strategy known as "tit-for-tat." "Tit-for-tat" is a strategy made famous by Axelrod's (1984) classic work on the repeated prisoner's dilemma game, where it was demonstrated that this "nice" (it is never the first to "defect") strategy does remarkably well against a wide variety of strategies. If "titfor-tat" is selected, the virtual partner will select Option A for the first trial and thereafter will select the same response that the participant had selected the previous trial. Thus, if the participant selected Option B for the first trial, the virtual partner programmed to play tit-for-tat will select Option B for the second trial, and so on, mirroring the behavior of the participant by selecting Option A if the participant selected Option A the previous trial, and retaliating "tit-for-tat" by selecting Option B if the participant selected Option B the previous trial. If the experimenter indicates Strategy 3 in the fourth line of the PMX file (see Figure 5), this generates a contingent strategy known as "tat-for-tit." If "tat-for-tit" is selected, the virtual partner will select Option B for the first trial and thereafter will 


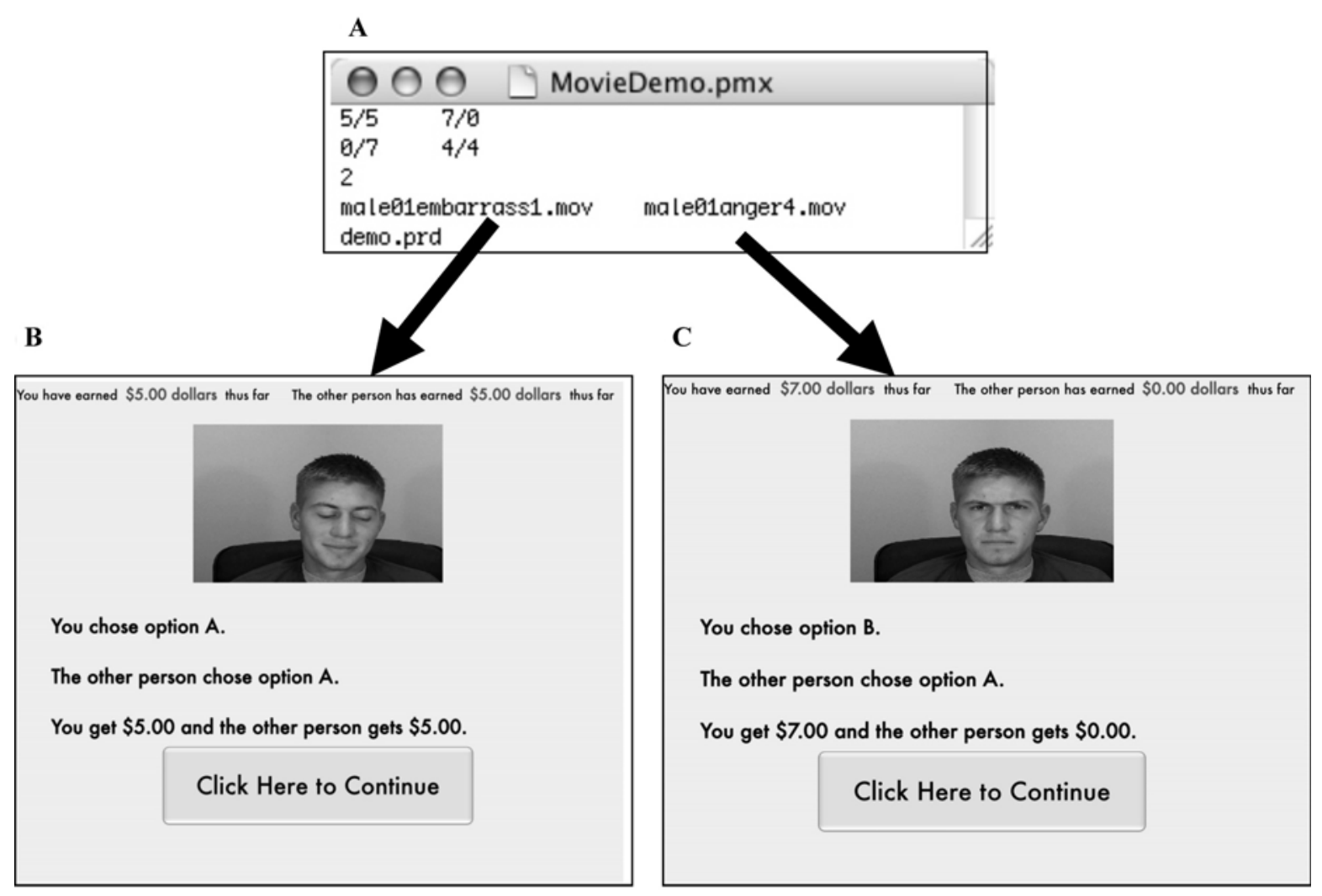

Figure 5. Screen captures depicting two different emotional signals displayed by the virtual interaction partner.

select a response that is the opposite of the response that the participant had selected the previous trial.

\section{Utilizing Prediction Screens}

Prediction screens are an optional feature of EMOTLAB that allows you to periodically query the research participants regarding the behavior and personality of their virtual partner. When the fifth line of text in a PMX file contains the name of a prediction screen file (see Figure 3 ) then a prediction screen will appear immediately after the results screen for that particular trial. Otherwise, if this line of text contains a zero, no prediction screen will appear for this particular trial. Prediction screens are generated from a prediction screen file. All prediction screen file names must end with the extension ".prd" and are stored in the Prediction Screens folder located inside the main EMOTLAB folder.

A prediction screen file generates a prediction screen that consists of two sets of questions. A first set of three questions appears on every prediction screen (see Figure 6) and cannot be edited. These three questions ask the participant to predict the behavior of their virtual partner during the next several trials (see Figure 6). The second set of questions on a prediction screen ask the participant to rate their virtual interaction partner on a number of adjectives. This second set of questions can be modified by the researcher by simply editing the list of adjectives that appear in the corresponding prediction screen file. For example, if a PMX file lists "demo.prd" in the fifth line of the file (see Figure 3), this prediction screen (which was created for EMOTLAB version 1.0) will appear immediately after the trial (see Figure 6).

\section{BASIC PROCEDURES FOR A TYPICAL EMOTLAB EXPERIMENT}

A typical EMOTLAB experiment entails creating several game list files, one for each experimental condition. A game list file is an editable text file that specifies the number of trials that the participant will encounter and lists the specific PMX files that generate each trial within a given round of play (see Figure 3 ). Game list files can be created from scratch or by simply copying and editing an existing file in the same way that one creates or edits PMX files. EMOTLAB Version 1.0 is distributed with two game list files: "MovieDemo.gml" and "PhotoDemo.gml."

Once the appropriate game list files have been created, the researcher can begin an experimental session by clicking on the appropriate EMOTLAB executable file (Windows or Mac OS X) that brings up the EMOTLAB Experimenter Setup Screen (see Figure 7). To assign a research participant to a specific experimental condition, the researcher selects the appropriate game file from the Game List pull-down menu (see Figure 7, "Round 1 [2 and 3] Condition" pull-down menus). All game list files end with the ".gml" extension, which is an abbrevation for "gamelist" and must be stored in the Game List Directory folder located within the main EMOTLAB folder. 


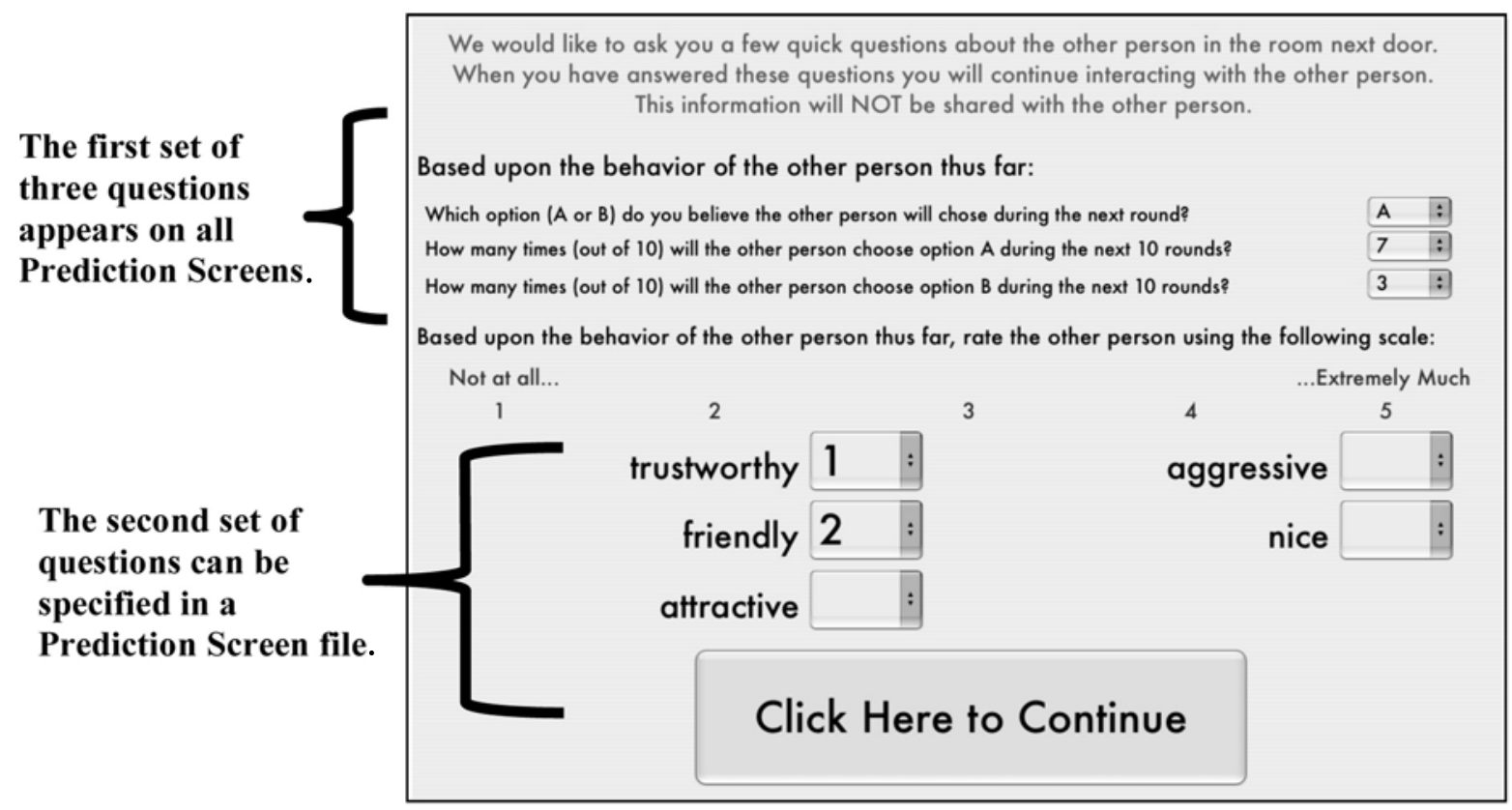

Figure 6. A sample prediction screen.

In addition to selecting up to three different game list files, the researcher also has the option of presenting a series of practice trials by selecting the appropriate Test List file from the Game List "Practice Rounds" pulldown menu (see Figure 7). Finally, the researcher can also specify the appearance of various title screens designed to present participants with additional information or instruction during the course of the experiment. A brief overview of the practice trial and title screen features is presented below.

\section{ADDITIONAL EDITABLE FEATURES OF EMOTLAB}

\section{Practice Trials}

Practice trials are an optional feature of EMOTLAB that introduces participants to the rules of the game (e.g., payoff structure) and then provides them with a brief series of quizzes (or tests) covering the rules and protocol of play. Only after participants have correctly answered these quiz questions are they allowed to continue to the practice trial. During the practice trial, participants practice making a selection (Option A or Option B) and learn how to view the digital image of their virtual partner during each trial (see Figure 1). After completing the practice trial the computer screen announces that participants will begin the first trial of the experimental session.

\section{Title Screens}

Title screens are an optional feature of EMOTLAB that allows the researcher to present information to participants at different points in the experimental session. Title screens can be programmed to appear immediately prior to the practice rounds and just preceding any of the three possible "Rounds." In order to display a title screen you must select the appropriate file from the Title Screen pulldown menu on the Experimenter Setup screen (Figure 4). All title screen files must be stored inside the Title Screen folder located inside the main EMOTLAB folder.

\section{ADDITIONAL STANDARD (NONEDITABLE) FEATURES OF EMOTLAB SOFTWARE}

In addition to the optional features of EMOTLAB that can be modified by editing text files (e.g., practice trials, title screens, etc.), there are several additional standard (noneditable) features of EMOTLAB Version 1.0 that are automatically generated during each experimental session. These features include: (1) an informed consent screen, (2) a demographics questionnaire screen, and (3) a social motives questionnaire screen.

The informed consent screen appears as the first screen after the researcher has completed the experimenter setup screen (Figure 7). It is assumed that EMOTLAB software will be used in an ethical manner in which the rights of research participants are protected. Thus, the informed consent screen welcomes participants to the experiment and reminds them to read and sign the informed consent form (this must be provided by the researcher) before proceeding. Only after participants have clicked on a button labeled "I have read the informed consent and wish to continue with the experiment" are they allowed to proceed to the demographics questionnaire screen. A sample of a separate video usage consent form is included on the website for researchers who wish to capture video of participants' behavior in these virtual interactions.

The demographics questionnaire asks participants to provide three pieces of information: (1) gender, (2) ethnicity, and (3) year of birth (used to determine age). Participants provide answers to these questions by selecting 


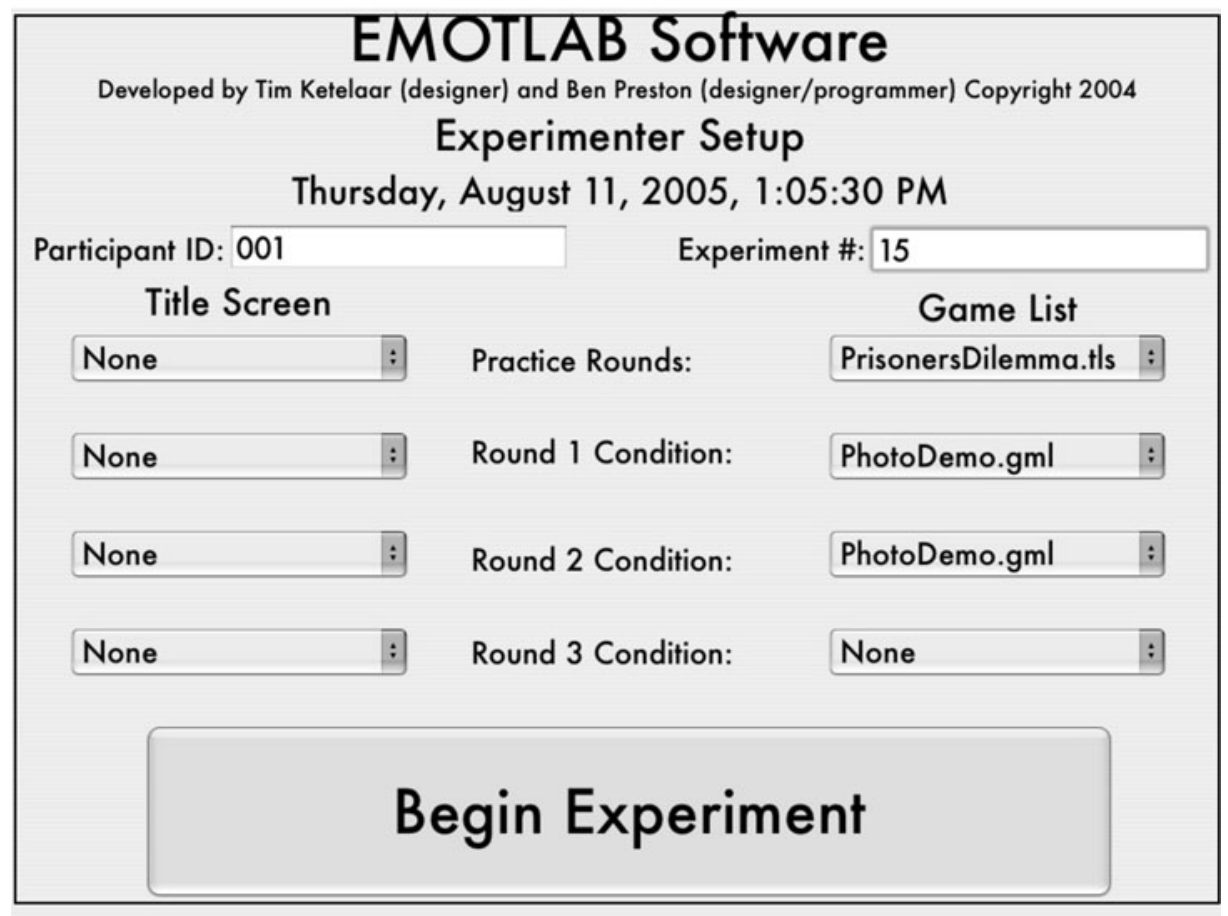

Figure 7. The EMOTLAB Experimenter Setup Screen.

the appropriate response option from a series of pull-down menus.

After completing the demographics questionnaire screen, participants are presented with a social motives questionnaire. This brief questionnaire must be completed before participants can proceed to the experimental trials (or practice trials, if that option is utilized). The social motives questionnaire is a nine-item measure of individual differences in preferences for resource distributions (Messick \& McClintock, 1968; Van Lange, Otten, DeBruin, \& Joireman, 1997). Responses on the social motives questionnaire can be utilized to sort participants into three stable strategy types: (1) cooperators, who routinely prefer outcomes that maximize joint payoffs between participants; (2) individualists, who routinely prefer outcomes that maximize their own payoffs with little regard for the payoffs that others receive; and (3) competitors, who prefer outcomes that maximize their relative advantage over their rival (Au \& Kwong, 2001; Van Lange, Otten, De Bruin, \& Joireman, 1997; Van Lange $\&$ Visser, 1999). Scoring instructions for the social motives questionnaire are contained in Appendix A (adapted from Van Lange \& Kuhlman, 1994) and are available on the website.

\section{WORKING WITH DATA FILES GENERATED BY EMOTLAB}

Each EMOTLAB session generates data output in the form of a text file stored in the Experimenter Data folder. These text (txt) files can be converted into a format (e.g., Excel) suitable for importing into a statistical software package. To import an EMOTLAB data file into Excel, for example, the researcher must open the software into which the data will be imported (e.g., Excel) and follow the procedures for importing an external text file. The data are stored as comma- and tab-delimited strings. A list of the variables generated by a single EMOTLAB session and a list of suggested variable names are provided in Appendix B and are available on the website.

\section{Availability}

EMOTLAB Version 1.0 operates on both the Windows and Mac OS X platforms and is available for download, at no cost, from www.psych.nmsu.edu/ ketelaar/emotlab .htm or by contacting the first author directly (ketelaar@ nmsu.edu). Additional instructions for operating EMOT$\mathrm{LAB}$ and using the various features of the program are available on the website at no charge.

\section{AUTHOR NOTE}

This research was supported, in part, by an NMSU minigrant, Studying Emotional Signals in a Virtual Social Interaction, awarded to T.K. The authors thank Matt Euler, Peter Jonason, Bryan Koenig, Tammy Calvano, and members of the Engineering Psychology program in the Department of Psychology at New Mexico State University for their assistance and helpful comments during the development of this software. Correspondence concerning this article should be addressed to T. Ketelaar, Department of Psychology, MSC 3452, New Mexico State University, Las Cruces, NM 88003-8001 (e-mail: ketelaar@nmsu.edu).

\section{REFERENCES}

Au, W. T., \& Kwong, J. Y. Y. (2002). Measurement and effects of social value orientation in social dilemmas: A review. In R. Suleieman, D. V. Budescu, I. Fischer, \& D. Messick (Eds.), Contemporary 
psychological research on social dilemmas (pp. 71-98). New York: Cambridge University Press.

AXELrod, R. (1984). The evolution of cooperation. New York: Basic Books.

Brown, W. M., \& Moore, C. (2002). Smile asymmetries and reputation as reliable indicators of likelihood to cooperate: An evolutionary analysis. Advances in Psychological Research, 11, 59-78.

Brown, W. M., Palmetta, B., \& Moore, C. (2003). Are there nonverbal cues to commitment? An exploratory study using the zeroacquaintance video presentation paradigm. Evolutionary Psychology, 1, 42-69.

CAMERER, C. F. (2003). Behavioral game theory: Experiments in strategic interaction. New York: Russell Sage.

Croson, R. [T. A.] (2006). Contrasting methods and comparative findings in psychology and economics. In D. de Cremer, M. Zeelenberg, \& J. K. Murnighan (Eds.) Social psychology and economics (pp. 301320). Mahwah, NJ: Erlbaum.

Davidson, R. J., Ekman, P., Saron, C., Senulius, J., \& Friesen, W. (1990). Approach-withdrawal and cerebral asymmetry: Emotional expression and brain physiology I. Journal of Personality \& Social Psychology, 58, 330-341.

de Cremer, D., Zeelenberg, M., \& Murnighan, J. K. (2006). Social psychology and economics. Mahwah, NJ: Erlbaum.

EKMAN, P. (2003). Emotions revealed: Recognizing faces and feelings to improve communication and emotional life. London: Weidenfeld \& Nicolson.

Ekman, P., Friesen, W. V., \& Hager, J. C. (2002). The facial action coding system (2nd ed.). London: Weidenfeld \& Nicolson.

ElsTER, J. (1995). Rationality and the emotions. Economic Journal, 116, 1386-1397.

ElSTER, J. (2000). Strong feelings: Emotion, addiction, and human behavior. Cambridge, MA: MIT Press.

Frank, M. G., \& EKMAN, P. (1993). Not all smiles are created equal: The differences between enjoyment and nonenjoyment smiles. Humor, $\mathbf{6}$, 9-26.

Frank, R. H. (1988). Passions within reason: The strategic role of the emotions. New York: Norton.

FRANK, R. H. (2004). Introducing moral emotions into models of rational choice. In A. S. R. Manstead, N. H. Frijda, \& A. H. Fischer (Eds.), Feelings and emotions: The Amsterdam symposium. New York: Cambridge University Press.

Frank, R. H., Gillovich, T., \& Regan, D. T. (1993). The evolution of one-shot cooperation: An experiment. Ethology \& Sociobiology, 14, 247-256

Gazzaniga, M. S., \& Smylie, C. S. (1990). Hemispheric mechanisms controlling voluntary and spontaneous smiling. Journal of Cognitive Neuroscience, 2, 239-245.

Guth, W., Schmittberger, R., \& Schwarze, B. (1982). An experimental analysis of ultimatum bargaining. Journal of Economic Behavior \& Organization, 3, 367-388

HiRSHLEIFER, J. (1987). On the emotions as guarantors of threats and promises. In J. Dupré (Ed.), The latest on the best: Essays on evolution and optimality (pp. 307-326). Boston: MIT Press, Bradford Books.

HIRSHLEIFER, J. (2001). Game-theoretic interpretations of commitment In R. M. Nesse (Ed.), Evolution and the capacity for commitment. New York: Russell Sage.

Ketelaar, T. (2006). The role of moral sentiments in economic decision making. In D. de Cremer, M. Zeelenberg, \& K. Murnighan (Eds.), Social psychology and economics (pp. 97-116). Mahwah, NJ: Erlbaum.

KetelaAr, T., \& Au, W. T. (2003). The effects of guilty feelings on the behavior of uncooperative individuals in repeated social bargaining games: An affect-as-information interpretation of the role of emotion in social interaction. Cognition \& Emotion, 17, 429-453.

Kirby, K. N., Petry, N. M., \& BicKel, W. K. (1999). Heroin addicts have higher discount rates for delayed rewards than non-drug-using controls. Journal of Experimental Psychology: General, 128, 78-87.

Messick, D. M., \& McClintock, C. G. (1968). Motivational basis of choice in experimental games. Journal of Experimental Social Psychology, 4, 1-25.

Poundstone, W. (1992). Prisoner's dilemma: John Von Neumann, game theory, and the puzzle of the bomb. New York: Anchor.

Scharleman, J. P. W., Eckel, C. C., Kacelnik, A., \& Wilson, R. K.
(1999). The value of a smile: Game theory with a human face. Journal of Economic Psychology, 22, 617-640.

Schelling, T. C. (1960). The strategy of conflict. Cambridge, MA: Harvard University Press.

Schelling, T. C. (1984). The intimate contest for self-command. In T. C. Shelling (Ed.), Choice and consequence: Perspectives of an errant economist. Cambridge, MA: Harvard University Press.

Searcy, W. A., \& Nowicki, S. (2003). The evolution of animal communication: Reliability and deception in signaling systems. Princeton, NJ: Princeton University Press.

Smith, A. (2000). The theory of moral sentiments. New York: Prometheus Books. (Original work published 1759)

VAn Lange, P. A. M., \& Kuhlman, D. M. (1994). Social value orientations and impressions of partner's honesty and intelligence: A test of the might versus morality effect. Journal of Personality \& Social Psychology, 67, 126-141.

Van Lange, P. A. M., Otten, W., DeBruin, E. M. N., \& Joireman, J. A. (1997). Development of prosocial, individualistic, and competitive orientations: Theory and preliminary evidence. Journal of Personality \& Social Psychology, 73, 733-746.

VAN LANGe, P. A. M., \& VISSER, K. (1999). Locomotion in social dilemmas: How people adapt to cooperative, tit-for-tat, and noncooperative partners. Journal of Personality \& Social Psychology, 77, 762-773.

Von Neumann, J., \& Morgenstern, O. (1944). Theory of games and economic behavior. Princeton: Princeton University Press.

Yamagishi, T., Tanida, S., Mashima, R., Shimoa, E., \& Kanazawa, S. (2003). You can judge a book by its cover: Evidence that cheaters may look different from cooperators. Evolution \& Human Behavior, 24, 290-301.

Zeelenberg., M., \& Beattie, J. (1997). Consequences of regret aversion 2: Additional evidence for effects of feedback on decision making. Organizational Behavior \& Human Decision Processes, 72, 63-78.

Zeelenberg., M., \& Pieters, R. (2006). Feeling is for doing: A pragmatic approach to the study of emotions in economic behavior. In D. de Cremer, M. Zeelenberg, \& K. Murnighan (Eds.), Social psychology and economics (pp. 117-140). Mahwah, NJ: Erlbaum.

\section{NOTES}

1. In these studies altruism was measured by a self-report questionnaire administered prior to the creation of the videotapes.

2. Involuntary smiles differ systematically from fake smiles (Frank \& Ekman, 1993; Davidson, Ekman, Saron, Senulius, \& Frisen, 1990; Gazzaniga \& Smylie, 1990). In particular, real happiness smiles involve contractions of both the obicularis oculi muscles (producing the telltale "crows feet" wrinkle associated with "smiling" eyes) and the zygomatic major (which turns the corners of the mouth upward into the characteristic smiling mouth display). Fake smiles, on the other hand, generally involve contractions of the zygomatic major only, tend to be less pronounced on the left side of the face (Gazzaniga \& Smylie, 1990), and display a shorter duration than true smiles (Frank \& Ekman, 1993).

3. In a typical extensive form "trust" game there are two players who move sequentially. The first player is presented with two possible first moves. Selecting one of the two options presents the second player with a choice of two subsequent options, one option benefits both players, and a second option benefits only the second player. However, if the first player chooses the alternative first move, the game ends immediately and the first player receives a small sum of the money that is considerably less than they might have obtained had they had allowed (i.e., trusted) the second player to make a move. In this manner, the strategy choice made by the first mover is considered to be a good indicator of whether the first player "trusts" the second player (Camerer, 2003).

4. Trained actors are often able to spontaneously produce realistic facial displays of emotion by simply recalling emotions and/or attempting to relive previous emotionally laden events. It is presumed that reliving emotions activates brain pathways associated with the facial motor nerve (cranial nerve seven). This technique of activating involuntary emotion displays by voluntarily reliving emotional experiences is often referred to as Stanislavski's Method, or simply, method acting.

5. The random delay is designed to be consistent with a protocol of play in which participants move simultaneously. In game theory "simul- 
taneity" refers to information states rather than temporal states. Thus, as long as neither participant, when selecting their moves, is aware of their partner's choice, their actions are regarded as simultaneous (see Hirschleifer, 2001).

6. The fields of psychology and economics often differ dramatically in their conventions regarding the appropriateness of employing deceptive cover stories in research (see Croson, 2006). EMOTLAB is designed to allow researchers to study emotional signaling without using deception. Researchers who wish to utilize deceptive cover stories to give the illusion of a "real person-real time" social interaction should consult their local ethics committee or institutional review board regarding the ethical issues involved with using deception in research activities.

7. EMOTLAB version 1.0 comes with several distinct digital photos for each emotion display for each of the virtual partners. There is currently only one version of each digital movie depicting each emotion display in EMOTLAB version 1.0. We are currently developing several versions of each digital movie for each emotion for both virtual partners. As these additional image files become available they will be posted to the website.
8. EMOTLAB includes several features that inform participants in regards to the monetary payoffs. First, just prior to beginning the first round of play participants are informed, via a series of computer screens, that the monetary payoffs that they receive are dependent not only on which option they (the research participant) select, but also on which option their interaction partner selects. At this time participants are also informed that a roll of the dice - conducted at the end of the experiment - will determine whether they will actually receive the money that they have earned during the experiment. Specifically, participants are informed that they will need to roll two 1s (1:36 odds) in order to receive the sum of money that they have earned. Previous research has demonstrated that this water-down incentive protocol (payoffs paid out with a probably less than 1.0) generates decision behavior that is practically indistinguishable from an incentive structure in which participants actually receive a monetary payout every trial (see Kirby, Petry, \& Bickel, 1999). Finally, after each round of play a results screen displays the participant's monetary earnings for the current round of play as well as a running total of the money that they have earned thus far.

\section{APPENDIX A}

Categories of Social Motivations Into Which Participants Are Classified

\begin{tabular}{cccc}
\hline \multirow{2}{*}{$\begin{array}{c}\text { Social Motives } \\
\text { Question Number }\end{array}$} & \multicolumn{3}{c}{ Social Motives Category } \\
\cline { 2 - 4 } & Cooperate & Individualist & Competitor \\
\hline 1 & $\mathrm{c}$ & $\mathrm{b}$ & $\mathrm{a}$ \\
2 & $\mathrm{~b}$ & $\mathrm{a}$ & $\mathrm{c}$ \\
3 & $\mathrm{a}$ & $\mathrm{c}$ & $\mathrm{b}$ \\
4 & $\mathrm{c}$ & $\mathrm{b}$ & $\mathrm{a}$ \\
5 & $\mathrm{~b}$ & $\mathrm{a}$ & $\mathrm{c}$ \\
6 & $\mathrm{a}$ & $\mathrm{c}$ & $\mathrm{b}$ \\
7 & $\mathrm{a}$ & $\mathrm{b}$ & $\mathrm{a}$ \\
8 & $\mathrm{c}$ & $\mathrm{a}$ & $\mathrm{c}$ \\
9 & $\mathrm{~b}$ & $\mathrm{c}$ & $\mathrm{b}$ \\
\hline
\end{tabular}

Note-Participants are classified into one of the three categories of social motives (i.e., cooperator, individualist, competitor) if they make at least six of the nine choices consistent with that category (see Van Lange, Otten, De Bruin, \& Joireman, 1997).

\section{APPENDIX B \\ List of Variable Labels That Appear in Each EMOTLAB Data File}

A single experimental session generates a text file containing eight types of variables, as shown in Figure B1.

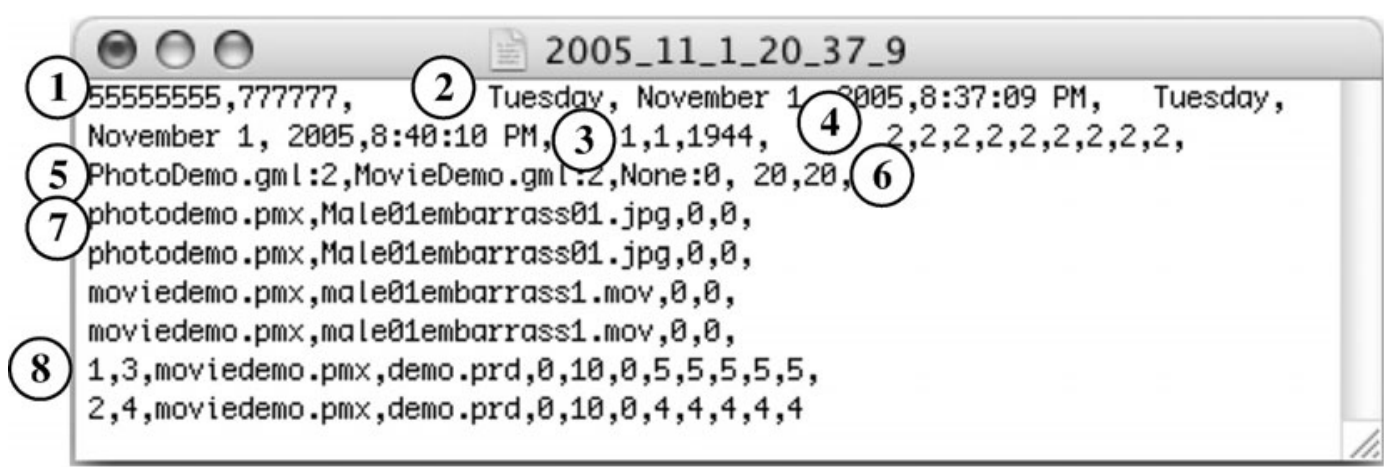

Figure B1. A typical EMOTLAB data file.

The following list provides suggested names for these variables, listed in the order that they appear (from left to right) in each Data file (see Figure B1). All variables are separated (delimited) by a comma, a semicolon, or a blank space. 


\section{APPENDIX B (Continued)}

\section{Participant and Experiment ID Variables}

These are the first two variables inputted (by the researcher) on the Experimenter Setup Screen

$\begin{array}{cl}\text { Suggested Name } & \text { Description } \\ \text { Id } & \text { participant id number } \\ \text { Expnum } & \text { experiment number }\end{array}$

\section{Start and Stop Time Variables}

These variables indicate the time/dates when the experimental session begins and ends. These variables are automatically recorded by EMOTLAB and do not require input from the researcher.

$\begin{array}{ll}\begin{array}{l}\text { Suggested Name } \\ \text { Dayin }\end{array} & \begin{array}{l}\text { Description } \\ \text { day in } \\ \text { month in } \\ \text { Monthin }\end{array} \\ \text { date in } \\ \text { Datein } & \text { year in } \\ \text { Yearin } & \text { time in } \\ \text { Timein } & \text { am or pm for start time } \\ \text { ampm1 } & \text { day out } \\ \text { dayout } & \text { month out } \\ \text { monthout } & \text { date out } \\ \text { dateout } & \text { year out } \\ \text { yearout } & \text { time out } \\ \text { timeout } & \text { am or pm for end time } \\ \text { ampm2 } & \end{array}$

\section{Demographic Variables}

These variables are automatically recorded when the participants answer the questions on the demographics page.

$\begin{array}{ll}\begin{array}{l}\text { Suggested Name } \\ \text { sex }\end{array} & \begin{array}{l}\text { Description } \\ \text { sex of participant: }(1=\text { Male; } 2=\text { Female) } \\ \text { ethnic }\end{array} \\ \text { ethnicity of participant: } \\ \text { (1= European American; } 2=\text { Latino; } \\ 3=\text { African American; } 4=\text { Asian American; } \\ \text { b= Native American; } 6=\text { Other) } \\ \text { birthyr } & \text { participant's birth year (range 1944 to 2004) }\end{array}$

\section{Social Motives Questionnaire Variables}

These variables are automatically recorded when the participant answers the nine questions on the Social Motives questionnaire page. See Van Lange and Kuhlman (1994) for complete scoring instructions.

\begin{tabular}{|c|c|}
\hline Sugoes & \\
\hline $\begin{array}{c}\text { Suggested } 1 \\
\text { sm } 1\end{array}$ & $\begin{array}{l}\text { Description } \\
\text { response to social motive questionnaire item } \# 1(1=\mathrm{A} ; 2=\mathrm{B} ; 3=\mathrm{C})\end{array}$ \\
\hline $\operatorname{sm} 2$ & response to social motive questionnaire item $\# 2(1=\mathrm{A} ; 2=\mathrm{B} ; 3=\mathrm{C})$ \\
\hline $\operatorname{sm} 3$ & response to social motive questionnaire item $\# 3(1=\mathrm{A} ; 2=\mathrm{B} ; 3=\mathrm{C})$ \\
\hline $\operatorname{sm} 4$ & response to social motive questionnaire item $\# 4(1=\mathrm{A} ; 2=\mathrm{B} ; 3=\mathrm{C})$ \\
\hline $\operatorname{sm} 5$ & response to social motive questionnaire item $\# 5(1=\mathrm{A} ; 2=\mathrm{B} ; 3=\mathrm{C})$ \\
\hline $\operatorname{sm} 6$ & response to social motive questionnaire item $\# 6(1=\mathrm{A} ; 2=\mathrm{B} ; 3=\mathrm{C})$ \\
\hline $\operatorname{sm} 7$ & response to social motive questionnaire item $\# 7(1=\mathrm{A} ; 2=\mathrm{B} ; 3=\mathrm{C})$ \\
\hline $\operatorname{sm} 8$ & response to social motive questionnaire item $\# 8(1=\mathrm{A} ; 2=\mathrm{B} ; 3=\mathrm{C})$ \\
\hline $\operatorname{sm} 9$ & response to social motive questionnaire item $\# 9(1=\mathrm{A} ; 2=\mathrm{B} ; 3=\mathrm{C})$ \\
\hline
\end{tabular}

\section{Experimental Condition Variables}

These variables identify the specific Game Files that are selected by the experimenter on Experimenter Setup Screen. Only the Game files selected for the Experimental conditions (Rounds 1-3) will appear in the data file, otherwise the word "none" will appear in the data file.

\section{Suggested Name round 1}

round $1 n$

round 2

round $2 \mathrm{n}$

round3

\section{Description}

Name of the Game List File selected for Round 1 Condition ("none" if no file is selected)

Number of the Payoff Matrix (PMX) Files executed by this Game File ( $=0$ if no Game file selected) Note: This number appears after the colon following the name of the game file.

Name of the Game List File selected for Round 2 Condition ("none" if no file is selected)

Number of the Payoff Matrix (PMX) Files executed by this Game File (=0 if no Game file selected) Note: This number appears after the colon following the name of the game file.

Name of the Game List File selected for Round 3 Condition ("none" if no file is selected) 


\section{APPENDIX B (Continued)}

round $3 n$

Number of the Payoff Matrix (PMX) Files executed by this Game File ( $=0$ if no Game file selected) Note: This number appears after the colon following the name of the game file.

\section{Total Monetary Earning Variables}

These variables identify the total amount of money that each participant has earned. These are the amounts that appear on the final screen after the participant has completed all trials. These variables are automatically recorded during an experimental session.

\begin{tabular}{ll} 
Suggested Name & $\begin{array}{l}\text { Description } \\
\text { earning }\end{array}$ \\
pearning & Total amount of money that participant has earned \\
\hline
\end{tabular}

\section{Individual Trial Variables}

These variables identify the names of the Payoff Matrix (PMX) files that generate each trial, as well as identifying the strategic choices (Option A or B) of the participant and their virtual partner during each trial. The image file displayed by the virtual partner during each trial is also indicated. These variables are automatically recorded during an experimental session. Note: For every trial (PMX File) four variables are recorded as shown below (note: suggested variables names for just two trials are presented here). Appendix Figure A indicates that four trials were generated in that experimental session.

\begin{tabular}{|c|c|}
\hline Suggested Name & Description \\
\hline triall & Payoff matrix file (if any) that generated this trial (1) \\
\hline image1 & Image file that that appeared this trial (1) \\
\hline Resp1 & Participant's response this trial $(0=\mathrm{A} ; 1=\mathrm{B})$ \\
\hline VPResp1 & Virtual partner's response this trial $(0=\mathrm{A} ; 1=\mathrm{B})$ \\
\hline Trial2 & Payoff matrix file (if any) that generated this trial (2) \\
\hline Image 2 & Image file that that appeared this trial (2) \\
\hline Resp2 & Participant's response this trial $(0=\mathrm{A} ; 1=\mathrm{B})$ \\
\hline VPResp2 & Virtual partner's response this trial $(0=\mathrm{A} ; 1=\mathrm{B})$ \\
\hline
\end{tabular}

\section{Prediction Screen Variables}

These variables identify the names of any Payoff Matrix (PMX) files that generated a prediction screen during the experiment, as well as a summary of the participant's predictions about their virtual partner's behavior and a set of optional trait ratings. These variables are recorded automatically recorded during an experimental session.

\section{Suggested Name}

PRDfile1

PRDfile1

PMXPRD1

PRDfile1

Nextroun

Oftena

Oftenb

Trait1

Trait2

\section{Description}

The number identifies the ordinal position of this Prediction screen file

(e.g., $1=$ this is the first prediction screen; $2=$ this is the second prediction screen) The number indicates the trial number during which this Prediction screen appears (e.g., $1=$ the first trial; $6=$ the sixth trial, etc.)

Name of Payoff Matrix (PMX) file containing this prediction screen

Name of first prediction screen file (located inside the PMX file)

Participant's prediction of what the partner will do in the next trial $(0=\mathrm{A} 1=\mathrm{B})$

Participant's prediction of how many times the partner will choose Option A during the next 10 trials (range $=0$ to 10 )

Participant's prediction of how many times the partner will choose Option B during the next 10 trials (range $=0$ to 10 )

Participant's rating of Trait2 (range $=1$ to 5)

Participant's rating of Trait3 (range $=1$ to 5 ) 